\title{
Sinderes
}

\section{Formación permanente de maestros en el Parque Explora: una aproximación a la comunidad Astromae'}

\author{
Permanent training for teachers of "Parque Explora" \\ An approach to the learning community Astromae
}

\begin{abstract}
Autores
Luisa Fernanda Casas Fernández ${ }^{2}$ Juan Pablo Suárez ${ }^{3}$

Recibido: 15 de noviembre de 2017. Aprobado: 10 de diciembre de 2017.
\end{abstract}

\footnotetext{
1 El presente artículo hace de los resultados parciales del proyecto de investigación titulado: Formación continua de maestros en escenarios diferentes a las facultades de educación: Un estudio de caso en relación a tres comunidades de Maestros Amigos de Explora -MAE-. Realizado entre 20162017 , en el marco de la práctica investigativa.

2 Licenciada en Educación Básica con énfasis en Humanidades y Lengua Castellana, del Tecnológico de Antioquia. luisa.casaso211@gmail.com.

3 Magister en Educación, docente ocasional y miembro del grupo Senderos del Tecnológico de Antioquia suarez7@tdea.edu.co.

4 Comunidad de maestros del parque explora que centran sus interese en temas de astronomía Astromae-
}

\section{Resumen}

El presente artículo está enmarcado en una investigación cualitativa Formación permanente de maestros en el Parque Explora: una aproximación a la comunidad Astromae, ${ }^{4}$ en el que se retomaron los discursos de los maestros con respecto a las percepciones que tienen de la comunidad Astromae referido al aporte a su práctica de enseñanza. Se recogieron los discursos de los maestros participantes, no participantes, y coordinadores de la comunidad mediante entrevistas semiestructuradas, las cuales permitieron reunir categorías que dan cuenta de unas características propias de conceptos teóricos como: formación permanente, comunidades de aprendizaje, trabajo colaborativo y práctica de enseñanza que aportan a los hallazgos y que sirven de soporte en la voz de los maestros en la discusión.

Palabras claves: Maestros, comunidades de aprendizaje, trabajo colaborativo, formación permanente, Astromae. 


\section{Abstract}

The article it is a cuantitative investigation called permanent formation for teachers in different places of the facultates of education a study of case in relation with the Teachers Friends of Explora-Astromae. At the one they will retake speaches by the teachers about the perceptions that they have about the Astromae.

In which teachers' discourses were take up again regarding their perceptions about Astromae community by means of semi-structured interviews. These enabled to gather categories that account for characteristics of an unconventional scenario, and subsequently, to understand the theoretical concepts such as permanent training, learning communities, collaborative work and teaching practice that contribute to the findings and will serve as a support to teachers' voice.

Keywords: Teachers, learning communities, collaborative work, teaching practice, permanent training, Astromae.

\section{Introducción}

La investigación de la cual se escribe el presente artículo, tuvo como pregunta ¿de qué manera la formación permanente de maestros en el Parque Explora: una aproximación a la comunidad Astromae aporta a la práctica de enseñanza de los maestros? Para empezar, es importante aclararle al lector que se encontrará con cuatro conceptos que corresponden a: formación permanente Vezud (2004) / Rodríguez Neira (1999) / Paz (2005), Comunidad de aprendizaje Krichesky \& Murillo Torrecilla (2011), Trabajo colaborativo Rodríguez \& Carlo Ossa (2014) Tardif (2004) / Henao (2013) Johnson, Johnson \& Holubec (1999) y práctica de enseñanza Olga Lucía Zuluaga Vasco (1997) los cuales son pertinentes para la comprensión de los resultados y la discusión. Además, es indispensable presentar una contextualización sobre la comunidad Astromae del Parque Explora, la cual es una comunidad de maestros que trabaja en torno a la astronomía, desde hace siete (7) años, se reúnen cada quince días (15) de manera voluntaria para generar intercambio de metodologías y actividades con respecto a este tema.

Para esta investigación se retomaron los discursos de ocho (8) maestros participantes, dos (2) maestros no participantes y tres (3) coordinadores de la comunidad, para responder a la pregunta ¿De qué manera Astromae aporta en la práctica de enseñanza de los maestros?, inicialmente se transcribieron las entrevistas, luego se analizaron para encontrar las relaciones para obtener las categorías de análisis. Después se propone una relación con la teoría planteada en el proyecto y la discusión entre ambas y se termina con las conclusiones del artículo con relación a los discursos presente en los participantes. 


\section{Aproximaciones teóricas:}

Para comenzar se retoma el concepto de formación permanente, el cual se da en escenarios no convencionales que recoge a la comunidad de aprendizaje Astromae, esta se concibió en el Parque Explora, un museo de ciencias y tecnología. Para ellos se presenta que la formación permanente desde (Amaya, Rosa. Amaya, Zoila., 2010) Cuando citan a (Rodríguez-Neira, 1999, pág. 1) así:

“[...] No se trata sólo de un perfeccionamiento teórico, académico o práctico,
sino de algo mucho más profundo y extenso, que abarca aspectos observables a
partir del advenimiento del paradigma posmoderno, cuyo talante sobresaliente
es la necesidad de que el aprendizaje se convierta en una actividad permanente".
(Rodríguez-Neira, 1999, pág. 1)

En este sentido, el profesor Rodríguez postula la formación permanente como un ejercicio de constante perfeccionamiento tanto teórico como práctico, así mediante la profundización de las dimensiones de aprendizaje de los maestros, lo que se quiere es lograr que la formación permanente se relacione como una necesidad que precise ser resuelta constantemente. Paz (2005) la define como:

Un proceso inherente a la práctica cotidiana de los sujetos que se desempeñan
como profesionales de la educación que promueve la autoformación, proceso
esencial por la potencialidad de generar los cambios y transformaciones en el
plano interno del sujeto y en el contexto donde ejerce su profesión. La formación
permanente no tiene como exigencia un programa prefijado, es un proceso que
depende más de las motivaciones intrínsecas de los docentes, que de lo pautado
externamente. (Paz, 2005)

Ahora bien, La profesora Paz menciona la existencia de tres elementos claves que componen la formación docente: el primero, es un proceso inherente al quehacer del maestro, el segundo que no tiene como exigencia un programa previamente establecido y estático como ocurre en la Universidad, y el tercero parte de las motivaciones intrínsecas de los maestros quienes dinamizan que la práctica de la formación permanente sea posible. Después de entender la formación permanente se propone el concepto de comunidad de aprendizaje. Dicho por Krichesky y Murillo Torrecilla, en el cual citan a (Mitchell y Sackney, 2000; Stoll, Bolam et al., 2006) "Desde una visión centrada en los profesores: la CPA puede definirse como un grupo de personas compartiendo e interrogándose críticamente sobre su práctica de modo continuo, reflexivo, colaborativo, inclusivo y orientado hacia el aprendizaje de los alumnos" (Krichesky \& Murillo Torrecilla, 2011, p.69). Desde esta perspectiva se pueden destacar varios componentes de una comunidad de aprendizaje, el primero hace referencia al objeto de estudio; que reúne a un grupo de personas con los mismos intereses, en este caso docentes, que buscan reflexionar a partir de un diálogo colaborativo, ligado a este, el segundo componente se refiere a la manera en que estos colectivos meditan sobre el objeto de estudio, lo cual se da a través del compartir de 
saberes y los cuestionamientos que se hacen al interior de las comunidades, con el fin de cumplir con el tercer elemento, mejorar el aprendizaje de los estudiantes, por medio de la transformación de su práctica de enseñanza.

En este sentido, para Krichesky \& Murillo Torrecilla (2011, p. 78) las comunidades de aprendizaje tienen diversos beneficios a destacar:

- Promueve el desarrollo profesional al recrear un ambiente que apoya el aprendizaje de los docentes, alentando a la innovación como solución alternativa a las problemáticas detectadas,

- Fomenta el trabajo grupal y la discusión, lo cual permite a los docentes revisar sus ideas previas o supuestos personales en un marco de intercambio.

A partir de lo anterior, una comunidad de aprendizaje permite el fortalecimiento de los vínculos entre los miembros que la conforman, a través de un diálogo constructivo de co-creación de nuevas posibilidades de práctica, tomando como factor relevante el trabajo colaborativo. Esto da muestra que dentro de las comunidades de aprendizaje existen roles que deben funcionar desde el aspecto colaborativo, en palabras del autor: "Favorecer el desarrollo de liderazgos compartidos [...] En esta idea, la dirección -el liderazgo formal- debe asumir un nuevo rol de mediador y dinamizador, más que de gestor o único responsable.”(Krichesky \& Murillo Torrecilla, 2011, p.77). En concordancia con esto se encuentra otro postulado:

La responsabilidad compartida, todos los miembros de la comunidad son participes en el proceso de aprendizaje. El conocimiento se entiende como dinámico, adquirirlo no supone ingerir una lista de elementos a reproducir en un examen, sino construir una comprensión propia de la materia. Es un proceso activo y colaborativo. (Kearney, p. 6)

Esto confirma la esencia vital de las comunidades de aprendizaje, en el trabajo colaborativo, el cual permite su buen funcionamiento, donde todos los participantes son actores activos dentro de su desarrollo profesional, y no simples receptores de información.

Sin embargo, no podría hablarse de comunidades de aprendizaje sin retomar el concepto de trabajo colaborativo y para ellos se retoma el trabajo de Felipe Rodríguez \& Carlo Ossa (2014), cuya propuesta investigativa plantea que:

El trabajo colaborativo es una de las principales estrategias organizacionales y curriculares utilizadas para el aprendizaje desde un enfoque inclusivo (Graden y Bauer, 1999; Moliner, 2008; Stainback y Stainback, 1999). Agregando que existe mucha evidencia sobre los beneficios del trabajo colaborativo para desarrollar una educación inclusiva, mejorar la calidad de los aprendizajes y favorecer la cooperación entre los profesores (Crameret al.,2010; Moliner, 2008, Stuart et al., 2006, Villa, Thousand\&Nevin, 2008). Citado por (Rodríguez Rojas, Felipe Francisco \& Ossa Cornejo, Carlos Javier, 2014, p. 19). 
Desde esta perspectiva el trabajo colaborativo es una estrategia que posibilita la organización de los docentes en relación al currículo con el objeto de propiciar el trabajo colaborativo entre ellos así, por tanto, favorece a que el saber docente se manifieste en las interacciones con otros y es en estas dinámicas donde se vinculan con lo que son, hacen, sienten, piensan y dicen. En este sentido el trabajo colaborativo posiciona al maestro como intelectual. Sin embargo, es pertinente precisar parafraseando Johnson, Johnson \& Holubec (1999): Que el aprendizaje colaborativo se estructura en cinco puntos básicos: Interdependencia positiva entre los miembros del grupo, Interacción cara a cara, Evaluación individualizada y responsabilidad personal para alcanzar los objetivos del grupo, Uso de las destrezas interpersonales y grupales, Evaluación constante.

Estos son aspectos claves en las dinámicas de aprendizaje y trabajo colaborativo entre docentes, a esto, Namo di Mello (2000) y Barrera Pedemonte (2008) agregan que se requiere una mínima estructura y de estímulos externos que permitan ir más allá de ser instancias de agradable intercambio, pero "vacías de contenido". Finalmente es importante vincular el concepto de práctica de enseñanza, el cual la enseñanza se convierte en el objeto de análisis de la pedagogía en tanto su desarrollo práctico o teórico como saber pedagógico en escenarios en los que se puede evidenciar las transformaciones de la práctica de enseñanza entendida como enseñanza, al respecto De Tezanos agrega:

"En relación con la noción de transformación de la práctica pedagógica,
entendida esta última como el lugar donde se inicia la construcción del saber
pedagógico, se hace necesario aclarar que si bien en toda práctica docente
es posible reconocer la presencia de principios teóricos que acompañan a la
tradición del enseñar, ésta asume modos de concreción diferentes según el
contexto socio-cultural en el cual opera." (2010, p 14)

Es decir, el maestro tiene un saber que pone en evidencia desde sus prácticas docentes o de enseñanza, pero estas prácticas están determinadas por un contexto y las relaciones que establezca con él. En este sentido Eloísa Vasco (1997) en "Maestros alumnos y saberes: investigación y docencia en el aula", plantea que el maestro cuando enseña establece la relación con cuatro preguntas que determina su accionar estas son: ¿Qué enseñar?, ¿Cómo enseñar?, ¿A quién enseñar? ¿Para qué enseñar?, con ellas es que el maestro establece el diálogo con el contexto, con los saberes a enseñar y con la pedagogía y otras disciplinas que le sirven de referencia para proponer la enseñanza.

Desde la perspectiva de Vasco (1997) el maestro en la práctica de enseñanza establece un diálogo entre el saber qué enseña, el sujeto a quién enseña y el cómo se le enseña, a esta perspectiva se suman (Jaramillo \& Gaitán, 2008), quienes coinciden con (Suriani, 2004), al señalar que las prácticas de enseñanza hacen referencia a los procesos de transmisión y apropiación de contenidos y saberes que se enmarcan en procesos de escolarización, los cuales se ponen de manifiesto en la relación docente-alumno- 
conocimiento, básicamente centrado en el enseñar y el aprender. Dichas prácticas tienen como especificidad el trabajo con el conocimiento y el cómo se comparte y se construye el mismo en las aulas. Adicional a esto (Maza, 2004, p.), argumenta que las prácticas de enseñanza implican:

- "Una actividad intencional y voluntaria en relación con el conocimiento, en un escenario específico; el aula

- Un complejo proceso de mediaciones

- Un juego y configuración mutua de hacer con teoría y teorizar sobre el hacer

- Un pensar y hacer en plural vinculados a decisiones éticas y políticas”

En este sentido se encuentra una relación dialógica que se debe establecer constantemente entre el quehacer del maestro en el aula y las bases epistemológicas que lo sustentan, es decir, el maestro debe estar en permanente diálogo con las teorías educativas y pedagógicas para cada vez tener una mejor comprensión de las mismas, fortaleciendo del mismo modo las competencias investigativas y favorecer la relación entre el maestro y la teoría y por ende, del maestro y el estudiante, llegando finalmente a procesos reflexivos que logren transformar las prácticas de enseñanza de los maestros.

\section{Metodología:}

La investigación cualitativa tiene como objeto “[...] el desarrollo de conceptos que ayuden a comprender los fenómenos sociales en medios naturales dando la importancia necesaria a las intenciones, experiencias y opiniones de todos los participantes." (Martínez Godínez, 2013, p. 5) y en este caso fue fundamental trabajar desde el enfoque fenomenológico - hermenéutico propuesto por Van Manen; donde "El valor fundamental de este tipo de investigación radica en su capacidad de acceder a la comprensión profunda de la experiencia humana investigada desde diversos ámbitos disciplinares."(Ayala Carabajo, 2008, p. 410) Esto significa la posibilidad de poder describir las experiencias tanto de los maestros participantes, no participantes y los líderes de (Astromae), para posteriormente analizarlos desde los métodos empleados para la recolección de información como la guía de entrevista. Para que por medio de esto haya una comprensión de las diversas formas en que los maestros expresan desde sus discursos el aporte que hace Astromae a su práctica de enseñanza.

Para comenzar, se les presentó a los participantes un consentimiento informado, en el que se cuida su identidad, para aplicar las entrevistas semiestructuradas. Se realizaron (14) preguntas a (8) maestros participantes en la comunidad Astromae quienes tenían las siguientes características: cinco (5) de los ocho (8) maestros participantes entrevistados son mujeres. Cuatro (4) de los maestros participantes trabajan con primaria, los demás trabajan en bachillerato. Cuatro (4) de los maestros participantes cuentan con maestría en educación. La edad de los maestros participantes oscila entre los 30 y los 50 años de edad. Siete (7) de los maestros participantes son solteros y dos (2) de los maestros tienen hijos. Las áreas de trabajo en las que se desempeñan 
los maestros corresponden a son: matemáticas, física, química y ciencias naturales. Todos los maestros participantes trabajan en instituciones de carácter público.

Estos maestros participantes llevan activos en Astromae un promedio de 3 años consecutivos, dando cuenta de la consistencia que tiene la red con respecto a sus participantes.

También se hicieron nueve (9) Preguntas a dos (2) maestros no participantes con las siguientes características: Ambos participantes trabajan en bachillerato en instituciones educativas de carácter público. La edad de los maestros no participantes oscila entre los 40 y 50 años de edad. Ambos maestros no participantes cuentan con maestría en educación. Uno (1) de los maestros no participantes es profesional no licenciado, el otro maestro (a) no participante es licenciado de la Normal Superior. Uno (1) de los maestros no participantes es soltero(a) y no tiene hijos, el otro maestro (a) no participante es casado (a) y tiene un hijo.

Además, se hicieron cinco (5) preguntas para los líderes de Astromae, (funcionarios del parque explora encargados de direccionar y coordinar las acciones de la comunidad). Ello tiene las siguientes características: dos (2) de los líderes poseen postgrado, uno (1) es bachiller, solo uno (1) de los líderes tiene formación académica en educación. La edad de los líderes oscila entre los 30 y los 45 años de edad. Dos (2) de los líderes son solteros(a), el otro líder está separado (a) y tiene un hijo. Las respuestas a estas preguntas se transcribieron en un cuadro en Excel, que permitió sacar frecuencias de palabras y posteriormente relaciones apoyadas por comentarios y descripción de la relación resultante.

Tabla 1 Frecuencias que sustenta la emergencia de las categorías

\begin{tabular}{|l|l|}
\hline \multicolumn{2}{|c|}{ Tabla de frecuencias } \\
\hline Categorías & Frecuencia de aparición en las entrevistas \\
\hline Astromae & 96 veces \\
\hline Astromae /motivación & 17 veces \\
\hline Astromae/ Integración de saberes & 8 veces \\
\hline Astromae / Metodología & 47 veces \\
\hline Red & 30 veces \\
\hline Comunidad & 10 veces \\
\hline
\end{tabular}

Fuente: elaboración propia 


\section{Resultados}

A partir del proceso de análisis de las respuestas a las entrevistas se determinaron como categorías aquellas palabras presentaron un alto número de apareció en los discursos de los participantes tal como se presenta en la tabla de frecuencias. En razón de lo anterior se determinaron las siguientes categorías para los resultados: Primera Categoría 1 y subcategorías: Astromae- motivación, Astromae- integración de saberes, Astromae- metodología y una segunda Categoría denominada Red y Comunidad. Las cuáles serán presentadas a continuación.

\section{Categoría 1: Astromae}

Esta categoría se consolida en cuanto fue la palabra por parte de los maestros participantes y los líderes de las comunidades que más referenciaron en su discurso encontrándose 96 veces nombrada. Con lo cual se logra concretar que Astromae es un espacio enfocado en maestros que trabajan en torno a la astronomía, pero que además permite la participación de otros profesionales interesados en este mismo tema. En este sentido los participantes coinciden en afirmar que es la astronomía el centro de interés que los une. Por consiguiente, en estos discursos se encontraron algunas relaciones que las tomaremos como subcategorías, estas son: Astromae- motivación, Astromae- integración de saberes, Astromae- metodología. Dichas relaciones se desarrollarán a continuación.

\section{Astromae/ motivación}

La consolidación de Astromae como categoría de análisis de la formación permanente de maestros pasa por reconocer que este tipo de formación pasa por la motivación, en este sentido, se encuentra que en la comunidad Astromae del parque explora, el vínculo que la hace funcionar esta mediado por la motivación lo cual se ratifica en los discursos de los ocho (8) maestros participantes, en este sentido uno de ellos dice: "los maestros trabajamos realmente seis horitas y media, siete... Y hay tiempo libre y uno lo dedica a lo que le gusta en este caso es matemáticas y astronomía” (MH, 2016, p. 4).

Esta afirmación, da cuenta del compromiso laboral del docente seis (6) y siete (7) horas diarias en su institución educativa; y de la dedicación a los temas de interés la astronomía, con lo cual encuentra en la comunidad un espacio propicio para desarrollar el gusto por ese tema en el tiempo libre que tiene por fuera de su jornada laboral.

Es esta una de las características más relevantes de la formación permanente que ofrece Astromae, se da en el tiempo libre. Tiempo que podría ser dedicado a otras acciones o actividades, sin embargo, el docente participante de Astromae lo hace por el gusto a la temática que los motiva a permanecer en esta comunidad.

De esta manera se puede afirmar que el maestro encuentra en Astromae una relación de motivación personal que lo conecta con su saber específico, convirtiéndose en 
un disfrute poder participar de la comunidad. Con lo cual, las dinámicas de las instituciones educativas en las que laboran no son impedimento para participar de la comunidad ya que los espacios de participación se dan en el tiempo libre del docente, lo que permite inferir la responsabilidad ética por su propia autoformación.

\section{Astromae/ Integración de saberes}

Esta relación emergió en los discursos de los maestros con menos intensidad que la anterior, sin embargo, ellos coinciden en afirmar que en la comunidad de Astromae se integra la astronomía con otros saberes como matemáticas, física, química, entre otras. Especificando que la astronomía puede ser un tema para integrar la materia que se desee, además la astronomía genera interés en los estudiantes ya que no es un tema muy desarrollado en el currículo.

En cuanto a la articulación de los saberes mediante la astronomía, es pertinente retomar las palabras de uno de los docentes participantes quien plantea “ [...] las matemáticas se relacionan con muchísimas áreas y mi gusto por astronomía, es porque a través de este tema (Astronomía) yo puedo enseñar mucho conceptos matemáticos y físicos entonces [...]”. (MH, 2016, p. 4). En esta reflexión se evidencia que el maestro puede integrar la astronomía con su área específica y cumplir sin complicación con el plan de estudios, es decir esta temática puede ser un recurso que aporta a las materias y conceptos propios del currículo.

Otro elemento encontrado que resalta la integración de saberes como característica de la comunidad Astromae, es la participación de personas de otras profesiones diferentes a los maestros, y la participación de profesores de distintas área, quienes aportan desde su profesión otras temáticas que se pueden tener en cuenta en el currículo escolar. Al respecto un maestro(a) participante comenta:

[...]Estamos integrados, hay profesores de todas las áreas, inclusive hay personas que son jubiladas y hay personal que no tiene nada que ver [con lo educativo] pero que les gusta y se metió a este cuento de astronomía después de haber venido una vez, dos veces o por comentarios de otros compañeros que han venido, pues esa es la diferencia que yo veo con otras redes porque en las otras redes si son profesores ya asociados a determinado saber. (MM, 2016, p. 2)

Lo anterior indica que la comunidad se convierte en un espacio de inclusión que no restringe la participación solo a maestros de matemáticas y física como se pensaría en tanto la cercanía temática, sino, que permite el intercambio de saberes entre diferentes profesionales que encuentran en la astronomía un interés que trasciende sus propios saberes y desde esta confluencia la comunidad Astromae logra una cohesión colectiva que los identifica. Ratificándose así que la astronomía es un tema que integra diferentes saberes en la comunidad. 


\section{Astromae / Metodología}

Esta relación, permite identificar dos aspectos diferentes, de un lado, a la metodología que se vive en los encuentros de la comunidad, de otro, la pertinencia en las Instituciones educativas y las aulas de clase de replicar o aplicar algunas acciones o ideas que se proponen en los encuentros. Por tanto para efectos de este artículo, se retomaran las iniciativas que docentes y líderes de la comunidad consideran pertinentes para desarrollar en las instituciones educativas.

En el discurso de los docentes y líderes de la comunidad Astromae, emergieron las siguientes propuestas o estrategias metodológicas: proyectos, laboratorios, semilleros de astronomía y actividades que apoyan conceptos desde la astronomía. Estas propuestas se orientaron hacia las instituciones educativas o las aulas de clase. A continuación, se describe cada una de ellas:

\section{Proyectos, desde la voz de uno de los maestros participantes se comprenden como:}

Los proyectos hacen parte de una estrategia en la que se vinculan todos los estudiantes de un mismo grupo o grado para llevar a cabo un producto final que está soportado por las demás asignaturas y en estos casos el tema central es la astronomía. (Entrevista 4- MMP- 2016)

Retomado esta explicación, se encuentra que los proyecto son estrategias de trabajo para el aula, la novedad está en centrar el proyecto a la astronomía, lo cual da cuenta de una apropiación por parte del maestro de la astronomía como pretexto de trabajo en el aula, apropiación que fue referenciada por (6) seis maestros participantes afirman que con la participación en Astromae han desembocado su trabajo en el aula a través de proyectos que tienen que ver con astronomía. A modo de ejemplo se retoma la experiencia de uno de los maestros participantes quien comenta: "Hace algún tiempo hice un proyecto con unos estudiantes de mi institución y de Argentina, se enviaban cartas y hablamos de mediciones con el sol y los solsticios, fue muy interesante" (MM, 2016, p. 4).

El testimonio de este maestro, da cuenta de la posibilidades que ofrece trabajar por proyectos y como el tema de la astronomía motiva a los estudiantes a trabajar en la escuela, de otro lado, que este maestro, logra trascender su quehacer docente y llevar a lso estudiantes a intercambiar experiencias con estudiantes de otro país, pero esto es posible gracias a la iniciativa del docente que de un lado participa de la comunidad en un proceso de formación permanente y de otro, establece vínculos con colegas de otros países. Y pone un poco más que otros docentes para que sus estudiantes puedan a través del currículo y las áreas propias del pensum académico, romper con el encierro de la escuela en tanto la comunicación mediante cartas con sus pares de otro país. 
Laboratorios: uno de los maestros explica: "Los laboratorios son diferentes actividades que aportan a una materia o conceptos específicos, se dan en dos o más momentos y pueden intervenir los estudiantes de un mismo grupo o grado". (MM, 2016) La estrategia de laboratorios es practicada por (2) dos maestros(a) quienes reconocen la importancia de incluir en el currículo escolar los temas de astronomía porque permiten transversalizar distintas materias, en uno de los maestros participante comenta:

"Yo lo articulo particularmente con los laboratorios que hago de ciencias, entonces yo tengo laboratorios de ciencias y cuando estamos en el tercer período y segundo período que toco temas del universo y lo que tiene que ver con el planeta tierra entonces hago laboratorios del sol, donde involucro los relojes de sol, los movimientos del sol, cuando vemos el planeta tierra y sus capas, cuando vemos los movimientos de la tierra entonces siempre hago un laboratorio experimental en el patio, lo hacemos con los modelos de planeta y los instrumentos que aprendía acá y hago más o menos unos siete laboratorios con ellos durante el año, pero relacionados al tema”. (MM, 2016, p. 3)

En este apartado, se da cuenta de la articulación que el maestro realiza entre lo trabajado en Astromae y los temas propios del currículo con lo cual logra a través de los laboratorios trascender la explicación pasiva del tema del universo y desarrollar los laboratorios que se han diseñado en la comunidad para acercar de mejor manera a los estudiantes a la comprensión del universo. En este caso los laboratorios obedecen a una materia general como "ciencias". Se constata también que los laboratorios son experimentales y no ocurren propiamente en el salón de clases, más bien se retoman otros espacios de la institución de manera práctica.

Semillero de Astronomía: en cuento a esta pro puesta uno de los maestros participante precisa: "Los semilleros de astronomía son espacios de encuentro extracurriculares en la que intervienen estudiantes de todos los grados convocados por un tema específico “astronomía” y parte del gusto de los estudiantes”. (ML, 2016, p. 2)

Desde la comunidad Astromae se encuentra que la promoción de los semilleros fue una iniciativa de uno (1) de los líderes quien coordinó los encuentros desde el parque explora, su propuesta se sustentaba replicar la metodología de la comunidad Astromae en este sentido se retoman las palabras del maestro líder quien comenta:

La idea inicial si es que lo que se hace en Astromae, los maestros lo repliquen en sus aulas de clase, con sus estudiantes, incluso yo les he insistido mucho para que conformen semillero en sus instituciones y practiquen todo lo que aprendemos en la comunidad, pero no todos lo hacen, muchos porque en sus instituciones no los dejan y otros porque aún no se atreven a hacerlo. (ML, 2016, p. 2) 
Sin embargo, y dadas las características del "semillero" se debe tener en cuenta lo siguiente: los semilleros se hacen de manera extracurricular, es decir en contra jornada académica con los estudiantes de la institución que deseen participar, además, en las instituciones educativas muchas veces no tiene los recursos para tener estudiantes "horas extra" en la institución, y otro factor es que los maestros en su tiempo libre, dedican a sus gustos y crear un semillero implicaría dedicar más de su tiempo en la institución donde labora. Pero, desde uno de los líderes de la comunidad los semilleros esta la manera más efectiva para llevar al aula la astronomía, sin embargo, solo (2) de los maestros ha logrado conformar semilleros en sus instituciones educativas.

Al respecto uno de los maestros participantes comenta: "Yo he tenido a lo largo de varios años semilleros de astronomía, me han funcionado mucho, tanto que hemos ganado varios premios ya. Así que yo podría decir que Explora me ha dado demasiado”. (MM, 2016, p. 4). Con este testimonio se constata que la estrategia de los semilleros permite generar un vínculo con el saber diferente al convencional, de otro lado, que esta estrategia depende del maestro, y se confirma que la astronomía es un tema generador que motiva tanto a docentes como estudiantes. Sin embargo, conformar y consolidar un semillero es una tarea adicional para el maestro y le implica otros tiempos adicionales a los de su jornada laboral, por tanto, quien lo realiza da muestras de su compromiso y motivación por llevar a los estudiantes a otras experiencias de formación, que sin duda son inspiradas por su propia experiencia en la comunidad Astromae.

Actividades astronomía: en la comunidad Astromae el líder, y los maestros participantes están siempre en búsqueda de proponer actividades que pueden apoyar temas específicos en las aulas clase de los maestros. De estas iniciativas se derivan acciones de validación de las propuestas por parte de los maestros participantes.

La validación se realiza en el aula de uno de los docentes para verificar con los estudiantes la pertinencia de la actividad, los resultados se socializan en el próximo encuentro de la comunidad Astromae y los maestros en conjunto retroalimentan, modifican y construyen más actividades. Al respecto un maestro participante afirma que:

En Astromae los maestros pueden equivocarse sin temor alguno, cuando se propone un instrumento o proyecto casi siempre alguno lo hace y lo prueba y los demás le hacen mejoras o lo prueban después. Es interesante porque hay profes de todas las áreas y grados y eso hace que cada instrumento tenga mil formas de hacerse y con públicos distintos hacia el mismo fin, eso es muy bello". (MM, 2016, p. 5)

Este apartado confirma que la comunidad Astromae es un lugar de reflexión pedagógica en la que se diseñan y experimentan propuestas para dinamizar las aulas de los maestros articulando la astronomía a los temas u objetos de las áreas y grados en los que se desempeñan como docentes. Confirma también que los docentes participantes de la comunidad están en un proceso de formación permanente en torno a mejorar sus prácticas pedagógicas. 


\section{Categoría 2: Red y Comunidad}

En la entrevista realizada a los participantes, uno de los tópicos de análisis fue clarificar desde ellos las categorías red y comunidad, en este proceso se logró encontrar que para los participantes existen diferencias entre red y comunidad. A continuación, se presentan las concepciones que se tiene de cada una de ellas.

\section{Red}

Al retomar los discursos de los maestros participantes y no participantes a propósito de la categoría red se identifican las siguientes características:

- Las redes son por fuera de las instituciones educativas.

- Existen diferentes redes, casi sobre todas las materias hay redes.

- Las redes de maestros responden a indicadores.

- Las redes son propuestas por diferentes entidades.

- Las redes de maestros son valiosas porque permiten el intercambio de experiencias.

- La convocatoria que hacen para participar en las redes es a través de correo electrónico y de manera despersonalizada.

En el análisis sobre red los participantes reconocen que en la ciudad existen diferentes redes, tal como se evidencia en el siguiente relato: "Redes de maestros pues... Casi todas las áreas tienen sus redes, por ejemplo, está la red de español, la red de matemática, están los de investigadores en ADIDA, no pues hay cantidad de redes. (MNP, 2017, p. 1) En este apartado, el maestro no participante en Astromae muestra en su discurso el conocimiento de diferentes redes de maestros y refleja como característica que las redes giran en torno a un tema en particular, y en cada área específica existe una red. Algunos maestros encuentran que las redes, son promovidas por diferentes entidades y dependiendo de quién los promociones dependerá el interés de los maestros, de esta manera se logra ver en el siguiente testimonio:

Acá reenvían un correo que llega de manera genérica desde la alcaldía y uno recibe muchos correos, otra cosa es cuando ve, este se puede hacer hay profesores con cierto bagaje con cierto recorrido, de resto es un correo que uno ignora y casi no le veo la relevancia porque pueden ser mis prejuicios o escepticismo, es que hay veces que yo veo, llega un señor y firme, indicadores y es una capacitación y tal, así lo veo también una cosa muy sínica, pero también hay una gente que tiene eso en el corazón. (MNP, 2017, p. 2)

Este testimonio, advierte una problemática en la difusión de las redes a través de correos electrónicos genéricos y masivos. Para los maestros según el relato, las invitaciones deben ser personalizadas y con información relevante que dé cuenta de la seriedad y propósito de la red ya que desconfían de algunos procesos que no responde a redes propiamente dichas, con lo cual se pierde el interés en participar en diferentes convocatorias. 


\section{Comunidad}

Al referirse al término de comunidad o comunidades los maestros participantes, líderes y maestros no participantes de Astromae, identifican las siguientes características:

- Las comunidades tocan diferentes tópicos desde las diferentes áreas del conocimiento.

- Las comunidades se dan en instituciones educativas organizadas administrativamente.

- Las comunidades tienen como fin generar conocimiento.

- Las comunidades buscan modificar las prácticas de enseñanza para generar una manera diferente de ver la educación.

Respeto a estas características se retoman varios apartados de las entrevistas que las confirman, en cuento a cambiar las practicas un maestro (a) participante comenta: [...] Pienso que todas (comunidades) tienen un mismo fin que la educación que nosotros impartimos a los niños sea diferente a lo que estamos acostumbrados que es la parte catedrática y partir de diferentes formas de ver el mundo. (MM, 2016, p. 3)

Esta definición de comunidad le otorga un sentido pedagógico que se orienta a modificar las prácticas de enseñanza de los maestros participantes en las comunidades en este caso en Astromae.

En cuanto a la generación de conocimiento, un maestro (a) no participante de Astromae comenta:

[...] depende la comunidad a lo que se dedique, si es a investigar pues entonces el producto de esa investigación va a ser para el enriquecimiento profesional y laboral y otras comunidades que se ven mucho en las normales es más que todo estudiar pedagogía, compartir experiencias, construir conocimiento, entonces sí, si actualiza. (MNP, 2017, p. 1)

Las comunidades tienen como característica el enriquecimiento profesional, también busca compartir experiencias y la actualización de los maestros, sin embargo, al ser gestadas en la institución educativa, tienen en cuenta otros factores como la población, contexto de la institución, directivas, maestros.

En cuanto a las comunidades en las instituciones educativas uno de los maestros no participantes en Astromae comenta: “[...], en algunas instituciones ya muy organizadas, muy estructuradas administrativamente y en las normales existe las comunidades educativas o comunidades académicas dentro de la misma institución”. (MNP, 2017, p. 3). Este comentario, es relevante en tanto identifica que las comunidades académicas se pueden desarrollar en instituciones educativas con una estructura administrativa consolidada. 
En definitiva, desde las voces de los participantes, las comunidades son estructura en las que los maestros consolidan su formación permanente en torno a su quehacer procurando siempre producir conocimiento que les permita mejora su práctica pedagógica.

\section{Discusión}

Después analizar los resultados de las entrevistas en relación a la comunidad Astromae y las prácticas de enseñanza de los docentes se hace necesario establecer un diálogo con la teoría, con lo cual se encuentran aspectos que requieren ser discutidos para comprenderlos, para este caso se considera hacer énfasis en los conceptos de “Comunidad de aprendizaje” y "trabajo colaborativo".

Con relación a comunidad de aprendizaje, tal como se planteó en el marco conceptual al retomar a Krichesky \& Murillo Torrecilla, 2011 y Kearney se concluye que una comunidad de aprendizaje es un escenario construido colaborativamente por los docentes que la conforman, en el cual existen roles compartidos que permiten compartir, reflexionar y co-crear, en miras de transformar su práctica de enseñanza, además tiene como pilar fundamental promover el trabajo colaborativo entre sus participantes. Podría decirse que en el caso de Astromae, uno de esos elementos es contradictorio, es decir, el postulado propone que las comunidades deben tener una responsabilidad compartida y auto gestionarse, pero en el caso de la comunidad Astromae, existe un liderazgo o un coordinador propuesto por Parque Explora quien propone y dirige las sesiones de acuerdo con las necesidades y si se retoma lo mencionado anteriormente se expresa que es necesario tener un liderazgo compartido y determinar roles en la comunidad. Uno de los líderes expresa que hay una intención de estar alerta con las necesidades que refieren los maestros así: “[...]Lo que hacemos los líderes es estar atentos a esas necesidades y presentar los contenidos, entonces sí, siempre se ha hecho lo que los profes han querido".(ML, 2016), sin embargo, estar atentos a las necesidades, no necesariamente implica una construcción colectiva como se expresa que deben funcionar una comunidad de aprendizaje. Por lo anterior, es necesario que la comunidad Astromae retome este elemento para generar entre sus participantes:

- Autogestión

- Trabajo colaborativo

- Integración grupal

- Descentralización de la comunidad

- Más participación de maestros

Otro elemento que emerge es mirar las comunidades de aprendizaje como un espacio de construcción colectiva, pero no es el único espacio, por ejemplo, los maestros que no participan de ninguna comunidad, proponen "los espacios informales" para conversar y aprender otros temas que pueden aportar a su práctica pedagógica "Para resumir, conversando con los compañeros en la cafetería y es así como socializo y aprendo de ellos”.(MNP, 2017) y de esta manera informal, los maestros encuentran 
en la conversación con el otro una oportunidad para representar en sus discursos cambios pedagógicos en su práctica. De lo anterior podría expresarse que, si bien estos encuentros posibilitan la interacción, no hacen parte de la configuración de una comunidad de aprendizaje ya que como se había mencionado anteriormente se necesitan unos roles compartidos y un objetivo común que potencie el trabajo colaborativo, para evitar que el encuentro se quede en una conversación que no trasforma las prácticas.

El otro referente, trabajo colaborativo, para esta discusión se retoma a Rodríguez \& Ossa, 2014, quienes plantean que el trabajo colaborativo implica:

[...] coordinar el trabajo hacia metas comunes, compartir un sistema de creencias, demostrar paridad en los roles, utilizar un liderazgo distributivo y actuar en forma cooperativa (Villa, Thousand\&Nevin, 2008). Responde a una discusión profesional entre los co-enseñantes, lo cual releva la característica dialógica que presenta como base esta estrategia, siendo por ello un mecanismo participativo y necesario para la efectividad de la práctica docente. Citado por (Rodríguez Rojas, Felipe Francisco \& Ossa Cornejo, Carlos Javier, 2014, p. 19)

Como ya se expuso anteriormente, el trabajo colaborativo implica establecer metas comunes, en el caso de Astromae esto parece darse en tanto los maestros asisten a la comunidad de manera voluntaria, atraídos por la astronomía, temática común y de interés para ellos. Sin embargo, las metas propuestas por los líderes parecen ser unas y las de los docentes otras, ya que para uno de los líderes la intención de la comunidad sería que “[...] los maestros son validadores de los contenidos que nosotros tenemos acá”. (ML, 2016). De acuerdo con esta idea, se distorsiona el concepto de trabajo colaborativo, al pensar al maestro como un sujeto validador que utilizó para tal fin. Pero quedarse en esta idea desdibuja la esencia que los maestros le otorgan Astromae cuando la reconocen como comunidad de aprendizaje.

Ahora bien en cuanto al liderazgo distributivo que plantan Rodríguez \& Ossa, 2014, es necesario revisar la propuesta de la comunidad Astromae, porque esta recae en un alto porcentaje en quien coordina la comunidad, y si la intención es la validación de contenidos se puede perder la esencia de la comunidad, en tanto falta la confrontación con la intención de los docentes participantes, para lograr la discusión profesional entre los co-enseñantes, que es lo primordial en un trabajo colaborativo.

Otro elemento existente dentro del trabajo colaborativo implica una coordinación hacia metas comunes, y es notorio que en la comunidad Astromae, este elemento es tenido en cuenta en tanto los maestros realizan proyectos en conjunto, coordinan actividades y se presentan a eventos académicos.

De otro lado, la intención de la comunidad Astromae es replicar la comunidad en las instituciones educativas, sin embargo, seis (6) de los maestros participantes afirmaron que es complejo trabajar de manera colaborativa con los compañeros de sus 
instituciones, "En mi trabajo es complejo, porque no hay mucho interés, yo no lo he intentado”. (ENTREVISTA 8-MHP-p.3) Es decir, en las instituciones educativas donde laboran los maestros de la Comunidad Astromae los demás maestros no se interesan en conformar comunidades académicas en tanto implica trabajo colaborativo. En este sentido, los docentes que participan de Astromae buscan aliados y cooperación por fuera de la institución educativa.

\section{Conclusiones}

Se puede concluir que los maestros trabajan de manera colaborativa en la comunidad Astromae, sin embargo, encuentran dificultad para trabajar de esta manera en sus instituciones educativas, en este sentido no se puede atribuir a Astromae la responsabilidad de trabajar de manera colaborativa en las instituciones esto dependerá de la organización y las dinámicas de interacción entre los docentes en dichas instituciones.

Pertenecer a una comunidad de aprendizaje parte de la motivación del maestro por la temática o dinámica que en ella se dé, razón por la cual, no necesariamente todo lo que se trabaja en una comunidad se revierte en la práctica de enseñanza, ya sea porque los temas no lo permitan o porque los espacios y dinámicas institucionales tampoco lo favorezcan.

Astromae para configurarse como una comunidad de aprendizaje propiamente dicha, debe propiciar una dinámica de roles compartidos entre los participantes, que permita el trabajo colaborativo y liderazgo de los maestros, en este sentido se requiere reforzar el liderazgo distributivo en la comunidad para generar en sus participantes una voz y un voto con referencia a las sesiones que viven y experiencias que tienen en el aula.

\section{Referencias}

Amaya, R., Amaya, Z. (2010). Mediación de la cultura docente en los proyectos de formación permanente. Magis, Revista Internacional de Investigación en Educación, 135-146.

Ayala, R. (2008). La metodología fenomenológico-hermenéutica de M. Van Manen en el campo de la investigación educativa. Posibilidades y primeras experiencias. Revista de Investigación Educativa, 409-430.

BarreraPedemonte, F. (2008). El aprendizaje entre pares como estrategia de formación continua de profesores . Centro de Perfeccionamiento, Experimentación e Investigaciones Pedagógicas, CPEIP.

De Tezanos, A. (2007). Oficio de enseñar, saber pedagógico: relación fundante. En: revista Educación y ciudad. Bogotá IDEP 7-27

Jaramillo, J., \& Gaitán, C. (2008). Caracterización de prácticas de enseñanza universitaria. Revista Educación y Desarrollo Social - Bogotá, D.C., Colombia - Volumen 2- No. 2, 11-29. 
Krichesky, G., \& Murillo Torrecilla, J. (2011). Las comunidades profesionales de aprendizaje. Una estrategia de mejora para una nueva escuela. Revista Iberoamericana sobre Calidad, Eficacia y Cambio en Educación, 65-83.

Martínez, V. L (2013). Obtenido de http://www.pics.uson.mx/wp-content/ uploads/2013/10/7_Paradigmas_de_investigacion_2013.pdf

Martínez, I. \& Cabello, M.J. (2015). Oficinas pedagógicas en red como estrategia de mejora de la formación del profesorado en Mozambique. Revista Electrónica Interuniversitaria de Formación del Profesorado 18(3), 1-12.

Maza, M. (2004). Las prácticas de enseñanza: construcción y reconstrucción de los modos de pensar y hacer.

Suriani, B. (2004). Congreso Latinoamericano de Educación Superior en el siglo XXI.Las prácticas de la enseñanza en contextos de cambio: características, dilemas y tensiones. Las prácticas de enseñanza en contextos de cambio: características, dilemas y tensiones. San Luis, Argentina: Universidad Nacional.

Namo di Mello, G. (2000). Formaçao inicial de professores para a Educaçao Básica: Uma (re-)visão radical. Documento preparado para el Seminario sobre perspectiva de la educación, Unesco/OREALC.

Paz, I. (2005). El colectivo de año en la orientación educativa a los estudiantes de las carreras pedagógicas. Integra Educativa No 4 /(II) No. 1, 219-237.

Rodríguez, F., \&Ossa, C. (2014). Valoración del trabajo colaborativo entre profesores de escuelas básicas de Tomé, Chile. Estudios Pedagógicos XL, No 2: 303-319.

Rodríguez, T. (1999). Cambio educativo: presente y futuro: comunicaciones. VII Congreso Nacional de Teorías de la Educación. VII Congreso Nacional de Teorías de la Educación.

Vasco, E. (1997). Maestros, alumnos y saberes: Investigación y docencia en el aula. Bogotá: Magisterio

Vezud, L. (2004). Hacia una pedagogía del desarrollo profesional docente. Modelos de formación continua y necesidades formativas de los profesores. 